\title{
Analysis Docking Of Plasmodium Falciparum Enoyl Acyl Carrier Protein Reductase (Pfenr) With Organic Compunds From Virtual Screening Of Herbal Database
}

\author{
Nya Daniaty Malau ${ }^{1 *}$, St Fatimah Azzahra ${ }^{2}$ \\ ${ }^{1}$ Department of Physics Education, Indonesia Christian University, Indonesia \\ ${ }^{2}$ Department of Chemistry Education, Indonesia Christian University, Indonesia \\ *Email: malaunyadaniaty@gmail.com; azzahraflh@yahoo.co.id
}

Received April 23, 2019; Accepted February 08, 2020

\begin{abstract}
Malaria is one of problematic infectious diseases worldwide. The absence of an effective vaccine and the spread of drug resistant strains of Plasmodium clearly indicate the necessity for the deveploment of new chemotherapeutic agents. Recent method being developed is searching a new drug of antimalarial using in silico screening, or also known as virtual screening. One of enzyme target that important for growth of the malaria parasite is Plasmodium falciparum Enoyl Acyl Carrier Protein Reductase (PfENR). Inhibition of this enzyme cause the fatty acid biosynthesis type II will be terminated. In this research, in silico screening was performed using AUTODOCK VINA software to find inhibitor candidates of PfENR by using ligands from the database of Medicinal Plants in Indonesia. On the AUTODOCK VINA software moleculer docking experiments were performed between ligands and macromolecule target PfENR. This target that has been optimized with residue removal and charges addition. Ligand is expected to be the PfENR inhibitors.
\end{abstract}

Keywords: malaria, docking, herbal database, drug

\section{INTRODUCTION}

Malaria is an infectious disease that can be found in almost all parts of the world, especially in tropical and sub-tropical countries. According to the World Health Organization (WHO), in 2012 there was a spread of malaria in 99 countries from 104 countries which in the WHO data, Indonesia was one of the 99 countries. According to the Indonesian Ministry of Health's Directorate of Animal-Based Disease Control, in 2012 there were 417,819 positive cases of malaria in Indonesia [1-4].

The efforts made to control and eradicate malaria have been many, however, the disease has developed again due to increased cases of resistance to some antimalarial drugs, vector resistance to insecticides, and human migration from one region to another [2]. More than $40 \%$ of the world's population lives in malaria endemic areas at risk of contracting malaria [3]. Malaria can directly cause anemia and can reduce work productivity. This disease is also still endemic in most parts of Indonesia. According to 2014 Ministry of Health data, the incidence of malaria in Indonesia in 2014 was
$1 \%$. The five provinces with the highest malaria incidence include Papua (29.57\%), West Papua $(20.85 \%)$, NTT $(12.81 \%)$, Maluku $(6.0 \%)$, and North Maluku (3.32 \%) [1].

Malaria is an erythrocyte infectious disease caused by protozoan parasites from the Plasmodium genus. The protozoa enters the human body through the intermediaries of the female Anopheles mosquito. There are four Plasmodium species which commonly infect humans, that is P. falciparum, P. vivax, P. ovale and $P$. malariae. Of the four species, the most common cause of severe disease and even death is $P$. falciparum, the disease is commonly called tropical malaria / cerebral malaria [5] while other species generally only cause fever and sometimes anemia $[2,6]$.

To overcome infections caused by plasmodium, it is necessary to provide safe, effective, practical and economically affordable anti-malaria drugs to reduce deaths from malaria. There are several malaria drugs that are developed by inhibiting or killing the asexual forms of malaria parasites in human erythrocytes, such as quinine, chloroquine, pyrimethamine, sulfonamides, sulfones and 
artemisinin derivatives. To overcome infections caused by plasmodium, it is necessary to provide safe, effective, practical and economically affordable anti-malaria drugs to reduce deaths from malaria. There are several malaria drugs that are developed by inhibiting or killing the asexual forms of malaria parasites in human erythrocytes, such as quinine, chloroquine, pyrimethamine, sulfonamides, sulfones and artemisinin derivatives. The problem is that lately the above drugs mostly fail in the healing process caused by parasites that become resistant to malaria drugs (Harijanto et al, 2009). Some suspected causes of malaria resistance to antimalarials, in this case chloroquine is a parasite does not have an active side to bind to chloroquine so that chloroquine malaria drugs cannot be concentrated in erythrocytes $[4,7]$.

Several enzymes that play an important role in the life cycle of plasmodium include haematin, hemozoin, plasmepsin, falsipain, falsicillin, dihydrofolate reductase, dihydroperoate synthase, Plasmodium falciparum Enoyl-ACP reductase (PfENR), and others. Inhibition of these enzymes is a precursor to the development of antimalarial drugs [8].

In the last decade, there have been potential targets for antimalarials. This target is the type II fatty acid biosynthesis pathway that takes place in Plasmodium falciparum, with a specific target of Plasmodium falciparum Enoyl Acyl Carrier Protein Reductase (PfENR). This target was chosen because it has an important role in the synthesis of fatty acids in the Plasmodium falciparum body [9].

For developing countries such as Indonesia, this clearly requires its own strategy so that the discovery of new drugs that are useful for the community can be achieved but not costly. These efforts are not only carried out by parties engaged in the economic sector, but also by scientists and technologists. Today there is an alternative way to find new drugs raised by computer experts and information technology. During this time the drugs were tested before being marketed using the in vitro and in vivo methods, whereas now the third method that is no less important is the in silico method or inside the computer. The use of computers in the discovery of new drugs aims to improve the efficiency of the simulation process and calculations in drug design. The computer offers an in silico method as a complement to in vitro and in vivo methods commonly used in drug discovery processes. The science that plays a role in this process is bioinformatics.
One branch of bioinformatics is in silico screening or in silico screening, which involves a database with relevant molecular structures that are tethered to the target protein. The assessment results are then used as identification of structures with potential further physiological bonds and activities that can be evaluated in experiments [10].

In this study, an analysis of the mechanism and interaction between Plasmodium falciparum Enoyl Acyl Carrier Protein Reductase (PfENR) and ligands will act as an inhibitor through the in silico study method. The ligand used was derived from the results of screening from the Plant Chemical Database that has be done before. Based on the results of in silico antimalarial screening using the Indonesian Medicinal Plant Database on the target enzyme Plasmodium falciparum Enoyl Acyl Carrier Protein Reductase (PfENR). These chemical compounds are Kaempferol 3-rhamnosyl- (1-3) rhamnosyl- (1-6) -glucoside or known as tea plants [11]. The compound is examined for its bonding ability with enzymes through bond-free energy $(\Delta G)$ and the resulting inhibition constant (Ki). The in silico method that will be carried out to examine these interactions is by molecular docking. This method is also used to save costs and time needed during research [11].

\section{METHODS}

The material used is a two-dimensional structure of the Kaempferol 3-rhamnosyl- (1-3) rhamnosyl- (1-6) -glucoside ligand or tea plant is known that you get online on the database https://pubchem.ncbi.nlm.nih.gov/. While the macromolecule file selected as the receptor is the enzyme Plasmodium falciparum Enoyl Acyl Carrier Protein Reductase (PfENR) with the code $1 \mathrm{NHG}$ which can be downloaded from the Bank Data Protein (GDP) database at the site http://www.rscb.org. The tool used consists of hardware and software. The hardware consists of a computer with the HP Pavilion dv2 laptop specifications (Amd Athlon neo dual core, 2GB ddr2 RAM, 250 seagate HDD, and VGA ati radeon $\times 1200)$. The software used consists of Autodock Vina, ADT 1.5.6 (The Scripps Research Institute, America, LigPlot +4.5 .3$ (Rowan Lawkowski), OS. Ubuntu 14.04 Its [1215].

\section{Receptor Identification}

The protein used as a receptor is the enzyme Plasmodium falciparum Enoyl Acyl Carrier Protein Reductase (PfENR) (GDP code: 1nhg) which was obtained online through the site 
http://www.rscb.org/pdb (Figure 1). The identification process was carried out using Discovery Studio Visualizer 4.0 software based on information obtained from the $1 \mathrm{nhg}$ coded Plasmodium falciparum Enoyl Acetyl Carrier Protein Reductase (PfENR) crystallization data conducted [14].

\section{D Receptor Structure Geometry Optimization}

The 1nhg-coded receptor contains the coordinates of all residual atoms, crystallographic structural factors and XRD experimental data from the Plasmodium falciparum Enoyl Acyl Carrier Protein Reductase (PfENR) enzyme so that it can determine the quality of the protein three-dimensional structure. The geometry optimization process was done with ADT 1.5.6 software. The initial stage was the removal of water molecules $(\mathrm{H} 2 \mathrm{O})$ around proteins, hetero atoms and natural ligands. Next was the addition of Gasteiger and Hydrogen charges. Files were stored in PDBQT format.

\section{D Ligand Geometry Optimization} The ligand that will be used is Kaempferol 3rhamnosyl- (1-3) -rhamnosyl- (1-6) -glucoside or known tea plants downloaded online at the database https://pubchem.ncbi.nlm.nih.gov/. The optimization process was done using ADT 1.5.6. The initial stage was nonpolar merged hydrogen gives Gasteiger charge and all files were stored in PDBQT format.

\section{Molecular docking process}

Grid Process and docking parameter validation are carried out with ADT 1.5.6, molecular docking is carried out with AutoDock Vina (Scripps Research Institute, USA) and it is assumed that all rotatable bonds (cyclic bonds) of ligands can rotate (flexible) and the receptor is fixed (rigid) (Morris et al. 1998). The following parameters are parameters that have been validated. The selected grid box size is $64 \times 52$ x $64 \AA$ with spacing centered $1,000 \AA$ at the receptor, center_x $x=46,572$, center_y $y=82,254$, center_z $=38,472$ and the box area covers the entire receptor structure. Exaustiveness is set to number 8 . The vina folder is placed on the $\mathrm{C}: \backslash$ Vina drive and then filled in the CONF.TXT file with the above parameters according to the center and size numbers. Execute the docking command using commands via the CMD window, call Vina.exe then type the command "C: I vina --config conf.txt --log log.txt", then press enter and wait for the process to complete. Ligands that have the smallest Gibbs free energy $(\Delta G)$ from the list are stored in PDBQT format (ADT 1.5.6) and converted to PDB format with DSV 4.0 [15].

\section{Analysis}

The docking results in the analysis of bond energy $(\Delta G)$, hydrogen bond, RMSD value, binding residue, using Ligplot +4.5 .3 , for $2 \mathrm{D}$ visualization using DSV 4.0 and ADT 1.5.6.

\section{RESULTS AND DISCUSSION}

\section{Receptor Identification}

Three-dimensional structure of the enzyme Plasmodium falciparum Enoyl Acyl Carrier Protein Reductase (PfENR) downloaded through the Protein Data Bank. Threedimensional structure of Plasmodium falciparum Enoyl Acyl Carrier Protein Reductase (PfENR) enzyme, from the results of crystallization carried out by Perozzo et al. (2002) coded PDB of $1 \mathrm{nh}$. Visualization of PDB 1nhg coded protein is done using DSV 4.0 software. The visualization results show that there are 4 chains, namely A, B, C and D and there are two natural ligands of TCL and NAD (Figure 1). Both natural ligands must be removed because the enzyme that will be docking must be a pure enzyme. Furthermore, the Ligan used is Kaempferol 3-rhamnosyl-(1-3) -rhamnosyl- (1-6) -glucoside or tea plant known.

\section{Bonding Energy (Gibbs free energy)}

Gibbs free energy ( $\Delta$ Gbinding) is a parameter of conformational stability between ligands and receptors. Thermodynamically the metabolic reactions in the body take place exergonically and endergonically. Exergonic reaction is a reaction that produces Gibbs free energy, which is the energy used to work at a fixed temperature and pressure. Exergonic reactions cause the free energy of the reagent molecules to fall, because their free energy is released during the reaction. Therefore, free energy products become lower than reagent free energy. The lower the free energy of a molecule, the more stable the molecule is and the reaction goes spontaneously. This is called thermodynamic equilibrium, the more negative the free energy the reaction will be more spontaneous or will quickly form a stable conformation [16]. The bonding energy of the molecular docking results for the Kaempferol 3rhamnosyl- (1-3) -rhamnosyl- (1-6) -glucoside compound produces a final value of $-12.4 \mathrm{kcal} /$ mol consisting of 9 ligand modes. This means that the Kaempferol 3-rhamnosyl- (1-3) - 
rhamnosyl- (1-6) -glucoside compound is stable to be used as an inhibitor of the enzyme Plasmodium falciparum Enoyl Acyl Carrier Protein Reductase (PfENR) because the lower

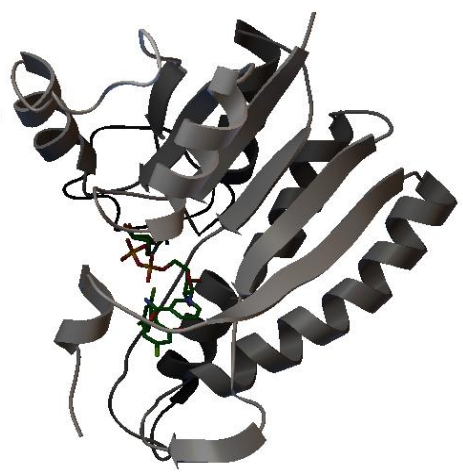

(a)

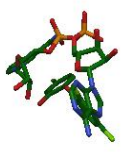

FIGURE 1. Visualization of 2D and 3D structures (a). Plasmodium falciparum receptor Enoyl Acyl Carrier Protein Reductase (PfENR) (b). Ligand of Kaempferol 3-rhamnosyl- (1-3) -rhamnosyl- (1-6) -glucoside compound

TABLE 1. The bond energy of the Ligan docking result

\begin{tabular}{cc|c|c}
\hline \multirow{2}{*}{ Mode } & \multirow{2}{*}{ Affinity (kcal/mol) } & \multicolumn{2}{c}{ dist from best mode } \\
\cline { 3 - 4 } & & $\begin{array}{c}\text { rmsd } \\
\text { I.b. }\end{array}$ & rmsd u.b \\
\hline 1 & -13.4 & 0.000 & 0.000 \\
2 & -13.3 & 2.141 & 8.775 \\
3 & -13.3 & 1.152 & 3.452 \\
4 & -13.3 & 2.788 & 9.052 \\
5 & -12.7 & 2.926 & 9.106 \\
6 & -12.7 & 2.603 & 8.862 \\
7 & -12.7 & 2.424 & 9.173 \\
8 & -12.4 & 1.973 & 4.318 \\
9 & -12.4 & 1.379 & 2.193 \\
\hline
\end{tabular}
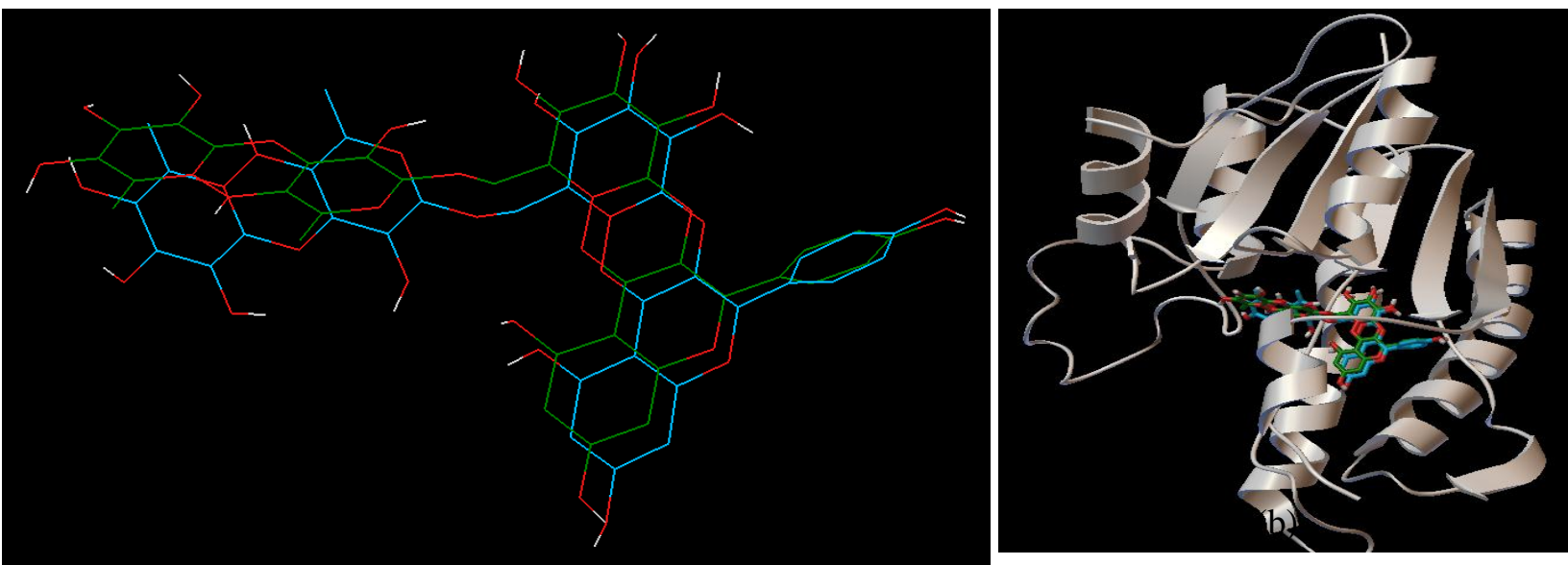

FIGURE 2. Pose Ligand compound Kaempferol 3-rhamnosyl- (1-3) -rhamnosyl- (1-6) -glucoside (a). Ligand Mode 2 and Mode 7 (b). Ligand Mode 2 and Mode 7 with reseptor

When viewed from the value of RMSD (Root Means Standart Deviation) the final value of RMSD from Kaempferol 3-rhamnosyl- (1-3) - rhamnosyl- (1-6) -glucoside is 1,375 Angstrom for RMSD 1.b and 2,193 Angstrom for RMSD u.b. This means that the Kaempferol 3- 
rhamnosyl- (1-3) -rhamnosyl- (1-6) -glucoside compound is stable to be used as a ligand because the RMSD value is below 2.5 Angstrom.

When viewed from the ligand obtained, two modes are most similar to the original ligand position and which is the mode with the best poses, mode 2 and mode 7 . Therefore, the compound Kaempferol 3-rhamnosyl- (1-3) rhamnosyl- (1-6) -glucoside is stable to be used as a ligand of the enzyme receptor Plasmodium falciparum Enoyl Acyl Carrier Protein Reductase (PfENR).

\section{Hydrogen Bond}

Hydrogen bonds are intermolecular attractions or between dipoles formed between two partial charges with opposite polarity. Hydrogen bonds occur when an atom gives its hydrogen covalent bond (donor) to an electronegative atom (acceptor), such as oxygen in -OH (Ser, Thr, Tyr, carbohydrate), $\mathrm{H} 2 \mathrm{O}$ and Nitrogen in -NH3 + (Lys, Arg) or -NH - (found in many peptide bonds, Trp, His, Arg, nucleotide bases) are all types of donors [17-18].

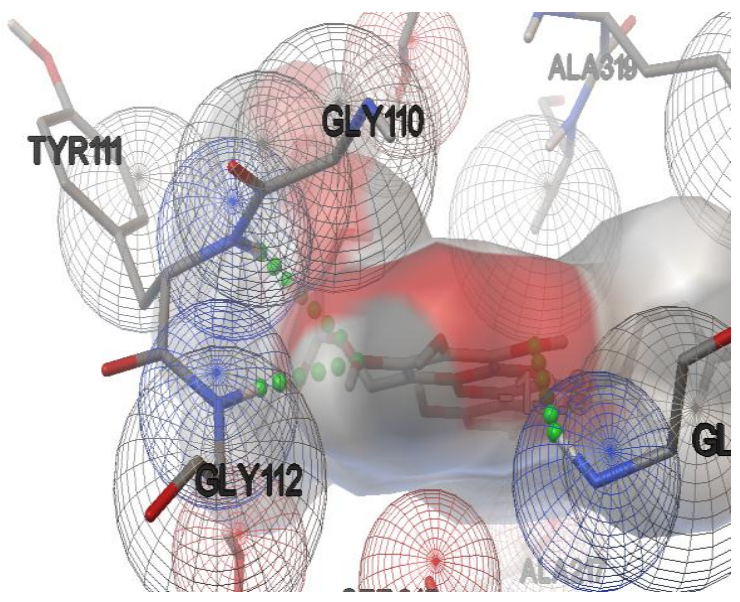

a)
Based on the hydrogen bond data obtained, then among the 9 ligand modes produced, mode 3 has the most hydrogen bonds, there are 3 hydrogen bonds that bind to the residues of Gly106, Tyr111, and Gly112. The next one that has a hydrogen bond 2 is mode 1 which is the hydrogen bond with a ligand and hydrogen bonds with a residue of Leu315. For ligands that have only 1 hydrogen bond present in mode 4 ligand which has a hydrogen bond at Ser317 residue, mode 5 has hydrogen bonds only at the residue Ala17 and mode 9 which has 1 hydrogen bond at the residue of Ala217. Whereas in mode 2, mode 6 , mode 7 and mode 8 do not have hydrogen bonds. The large number of hydrogen bonds determines the strength of the interaction. So that in ligand mode 3 is considered to have the highest stability between other fashion ligands. In addition, the overall ligand of Kaempferol 3rhamnosyl- (1-3) -rhamnosyl- (1-6) -glucoside is stable to be used as an inhibitor in the enzyme Plasmodium falciparum Enoyl Acyl Carrier Protein Reductase (PfENR) because there are hydrogen bonds that will increase stability.

FIGURE 3. Hydrogen bonds Ligand compounds Kaempferol 3-rhamnosyl- (1-3) -rhamnosyl- (1-6) glucoside (a). Ligan Mode 3 (b). Receptors and Ligand Mode 1

\section{Ligands Interaction and Receptors}

Hydrophobic interactions also play an important role in the stability of the ligand against the receptor. Hydrophobic interaction is an interaction that avoids the liquid environment and tends to group in the inner part of the globular protein structure to minimize interaction with water which can damage the structure of the protein and cause the enzyme to lose its activity [18-24]. In ligand mode 1, there are residues that form hydrophobic interactions, namely residues Leu315 and Ala217. From this interaction it can be seen that the ligand Kaempferol 3-rhamnosyl- (1-3) -rhamnosyl- (16 ) -glucoside is stable to be used as an inhibitor in the enzyme Plasmodium falciparum Enoyl Acyl Carrier Protein Reductase (PfENR) because there are hydrophobic interactions that will increase stability. The visualization of electrostatic interactions has not been able to be done by the software used in the study. But this interaction can be analyzed through residues that tend to form electrostatic 
interactions. Electrostatic interaction is an interaction between atoms caused by polarity. This interaction is a weak and non-covalent interaction so that it is easy to escape, but in large numbers, this interaction can have a large influence on stability [25-29]. Polar residues are charged at the receptor if the interaction with ligand mode 1 is observed, namely, Asp107. From this interaction it can be seen that the ligand of Kaempferol 3-rhamnosyl- (1-3) rhamnosyl- (1-6) -glucoside is stable to be used as an inhibitor in the enzyme Plasmodium falciparum Enoyl Acyl Carrier Protein Reductase (PfENR) because there are electrostatic interactions that will increase stability.

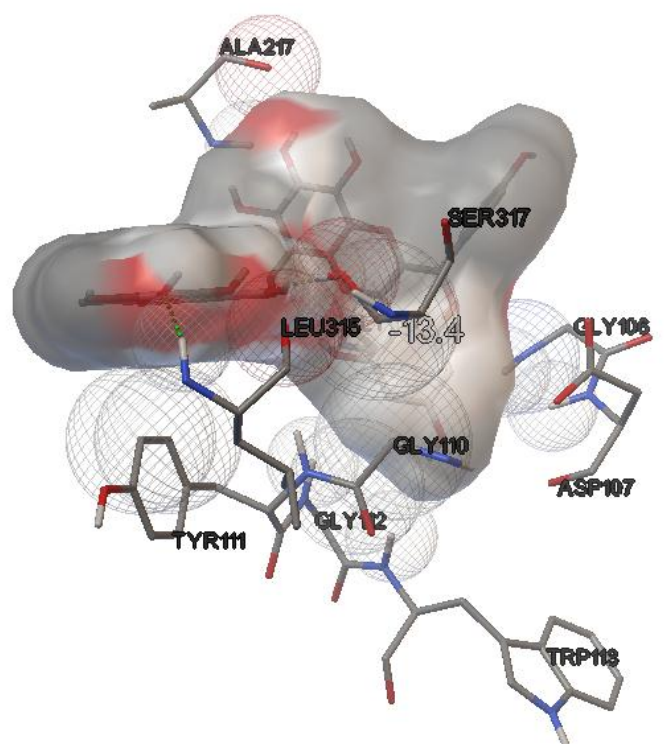

FIGURE 4. Interaksi Ligan mode 1 senyawa Kaempferol 3-rhamnosyl-(1-3)-rhamnosyl-(1-6)glucoside dengan reseptor Plasmodium falciparum Enoyl Acyl Carrier Protein Reductase (PFENR

\section{CONCLUSION}

Kaempferol 3-rhamnosyl- (1-3) -rhamnosyl(1-6) -glucoside compounds have good stability against the enzyme Plasmodium falciparum Enoyl Acyl Carrier Protein Reductase (PfENR. This is evident from the low bond energy, which is $-12.4 \mathrm{kcal} / \mathrm{mol}$ which consists of 9 ligand modes.This means that the Kaempferol 3rhamnosyl- (1-3) -rhamnosyl- (1-6) -glucoside compound is stable to be used as an inhibitor of the Plasmodium falciparum Enoyl Acyl Carrier Protein Reductase (PfENR) enzyme because it is increasingly low in free energy of a molecule, the molecule is more stable and the reaction runs spontaneously, besides that, Kaempferol 3-rhamnosyl- (1-3) -rhamnosyl- (1-6) -glucoside compound has a good bond between the ligand and the receptor stability of the enzyme Plasmodium falciparum Enoyl Acyl Carrier Protein Reductase (PfENR. The hydrogen bond formed is seen in three hydrogen bonds, namely at the residue of Gly106, Tyr111, and Gly112 in the 3 ligand mode. The interaction between ligands and receptors is also formed a stable is seen in the hydrophobic interactions seen at Leu315 and Ala217 residues and electrostatic interactions at Asp107 residues. From this interaction it can be seen that the ligand of Kaempferol 3-rhamnosyl- (1-3) -rhamnosyl- (16) -glucoside is stable to be used as an inhibitor in the enzyme Plasmodium falciparum Enoyl Acyl Carrier Protein Reductase (PfENR) because there are electrostatic interactions that will increase stability.

\section{ACKNOWLEDGMENTS}

The authors thanks to Research Computational Unit (RCU) Department of Physics Education FKIP UKI, for providing high performance computing to run all dynamics simulations. This research was supported by a research grant from Indonesia Christian University.

\section{References}

[1] Kementerian Kesehatan RI. (2011), Epidemiologi Malaria di Indonesia. Buletin Jendela Data dan Informasi Kesehatan. ISSN 2088 - 270X. Volume 1:1-39.

[2] Fauci, A. S. (Ed.). (2008). Harrison's principles of internal medicine (Vol. 2, pp. 1612-1615). New York: McGraw-Hill, Medical Publishing Division.

[3] Aguiar, A. C. C., da Rocha, E. M., de Souza, N. B., França, T. C., \& Krettli, A. U. (2012). New approaches in antimalarial drug discovery and development: a review. Memorias do Instituto Oswaldo Cruz, 107(7), 831-845.

[4] Dale, P., Sipe, N., Anto, S., Hutajulu, B., Ndoen, E., Papayungan, M., ... \& Prabowa, Y. T. (2005). Malaria in Indonesia: a summary of recent research into its environmental relationships. Southeast Asian Journal of Tropical Medicine \& Public Health, 36(1), 1-13. 
[5] Katzung, B. G. (2006). Cancer Chemotherapy. Basic and Clinical Pharmacology.

[6] Catteral, W. A., \& Goodman, M. K. (2006). Gildmans. The pharmacological basis of therapeutics 11 th edition. Chapter 14.

[7] Avery, M. A., Choi, S. R., \& Mukherjee, P. (2008). The fight against drug-resistant malaria: novel plasmodial targets and antimalarial drugs. Current medicinal chemistry, 15(2), 161-171.

[8] Sullivan, D., \& Krishna, S. (Eds.). (2006). Malaria: drugs, disease and postgenomic biology (Vol. 295). Springer Science \& Business Media.

[9] Tasdemir, D. (2006). Type II fatty acid biosynthesis, a new approach in antimalarial natural product discovery. Phytochemistry Reviews, 5(1), 99-108.

[10] Pripp, A. H. (2007). Docking and virtual screening of ACE inhibitory dipeptides. European Food Research and Technology, 225(3-4), 589-592.

[11] Wolff, M. E. (1996). Burger's medicinal chemistry and drug discovery. American Journal of Therapeutics, 3(8), 608.

[12] Adejoro, I. A., Waheed, S. O., \& Adeboye, O. O. (2016). Molecular docking studies of Lonchocarpus cyanescens triterpenoids as inhibitors for malaria. Journal of Physical Chemistry \& Biophysics, 6(2), 2161-0398.

[13] Hasan, M. A., Mazumder, M. H. H., Chowdhury, A. S., Datta, A., \& Khan, M. A. (2015). Molecular-docking study of malaria drug target enzyme transketolase in Plasmodium falciparum 3D7 portends the novel approach to its treatment. Source code for biology and medicine, 10(1), 7.

[14] Perozzo, R., Kuo, M., Valiyaveettil, J. T., Bittman, R., Jacobs, W. R., Fidock, D. A., \& Sacchettini, J. C. (2002). Structural elucidation of the specificity of the antibacterial agent triclosan for malarial enoyl acyl carrier protein reductase. Journal of Biological Chemistry, 277(15), 13106-13114.

[15] Morris, G. M., Goodsell, D. S., Halliday, R. S., Huey, R., Hart, W. E., Belew, R. K., \& Olson, A. J. (1998). Automated docking using a Lamarckian genetic algorithm and an empirical binding free energy function. Journal of computational chemistry, 19(14), 1639-1662.

[16] Cox, M. M., \& Nelson, D. L. (2008). Principles of biochemistry. WH Freeman \& Company.

[17] Philp, D., \& Robinson, J. M. (1998). A computational investigation of cooperativity in weakly hydrogen-bonded assemblies. Journal of the Chemical Society, Perkin Transactions 2, (7), 16431650.

[18] Lins, L., \& Brasseur, R. (1995). The hydrophobic effect in protein folding. The FASEB journal, 9(7), 535-540.

[19] Sharp, K. A., \& Honig, B. (1990). Electrostatic interactions in macromolecules: theory and applications. Annual review of biophysics and biophysical chemistry, 19(1), 301332.

[20] Hirjani, H., Mudasir, M., \& Pranowo, H. D. (2018). Prediction of High Performance Liquid Chromatography Retention Time for Some Organic Compounds Based on $\mathrm{Ab}$ initio QSPR Study. Acta Chimica Asiana, 1(1), 24-29.

[21] Adeoye, A. O., Olanlokun, J. O., Tijani, H., Lawal, S. O., Babarinde, C. O., Akinwole, M. T., \& Bewaji, C. O. (2019). Molecular docking analysis of apigenin and quercetin from ethylacetate fraction of Adansonia digitata with malariaassociated calcium transport protein: An in silico approach. Heliyon, 5(9), e02248.

[22] Singh, I. V., \& Mishra, S. (2019). Molecular docking studies of benzamide derivatives for PfDHODH inhibitor as potent antimalarial agent. Am $\mathrm{J}$ Biochem Molec Biol, 9, 1-6.

[23] Bhole, P., Lokhande, K. B., Nagar, S., \& Swamy, K. V. (2017). Molecular Docking 
and Molecular Dynamics Simulation studies of DHFR inhibitors in Plasmodium falciparum. Canadian Journal of Biotechnology, 1(Special), 23.

[24] Dohutia, C., Chetia, D., Gogoi, K., Bhattacharyya, D. R., \& Sarma, K. (2017). Molecular docking, synthesis and in vitro antimalarial evaluation of certain novel curcumin analogues. Brazilian Journal of Pharmaceutical Sciences, 53(4).

[25] Vijayaraghavan, S., \& Mahajan, S. (2017). Docking, synthesis and antimalarial activity of novel 4anilinoquinoline derivatives. Bioorganic \& medicinal chemistry letters, 27(8), 16931697.

[26] David, T. I., Adelakun, N. S., Omotuyi, O. I., Metibemu, D. S., \& Ekun, O. E. (2018). Molecular docking analysis of phytoconstituents from Cannabis sativa with pfDHFR. Bioinformation, 14(9), 574.

[27] Singh, S., \& Yadav, R. (2019). Homology modeling and docking study of Shewanella-like protein phosphatase involved in the development of ookinetes in Plasmodium. Journal of pharmacy \& bioallied sciences, 11(3), 223.

[28] Isea, R. (2018). A Point of View about Computational Challenges for the Design of New Drugs for Combating Malaria. Acta Scientific Microbiology, 1, 15-16.

[29] Khanal, P., Magadum, P., Patil, B. M., \& Hullatti, K. K. (2019). In silico Docking Study of Limonoids from Azadirachta indica with pfpk5: A Novel Target for Plasmodium falciparum. Indian Journal of Pharmaceutical Sciences, 81(2), 326332. 